\title{
一种量子化学组合方法及其应用于录对硫酸气溶胶形成影响的研究
}

\author{
李程桥王一波* \\ (贵州大学化学与化工学院 贵州省高性能计算化学重点实验室 贵阳 550025)
}

\begin{abstract}
摘要 本工作建立了一种量子化学的组合方法并应用于研究在硫和永复合污染地区, 录及其化合物是否会参与和驱动 硫酸气溶胶形成. 该方法采用 Molclus Genmer 模块生成数以百万计的巨量团簇初始结构, 用自洽紧束缚 GFN2-xTB 方 法预优化, 再用 B3PW91-D3(BJ) DFT 方法重新优化, 结合能经 $\omega$ B97M-D3(BJ)校正后计算出团簇的热力学函数, 以其 生成过程 Gibbs 自由能变大小排序, 获得团簇稳定序列及其对应的结合能和热力学函数. 然后用该方法研究了永及其 化合物、硫酸和水生成的 8 种团簇模型共计 240 万个结构, 结果表明 $\mathrm{HgSO}_{4}, \mathrm{HgO}$ 等录的极性化合物以及 $\mathrm{Hg}_{2}{ }^{2+}$ 和 $\mathrm{Hg}^{2}$ 能够促进硫酸气溶胶的形成, 而 $\mathrm{Hg}$ 以及无极性的 $\mathrm{HgCl}_{2}$ 和 $\mathrm{Hg}_{2} \mathrm{Cl}_{2}$ 对硫酸气溶胶成核的影响较小. 这一结论对硫、 永复合污染环境下，硫酸气溶胶成核机制探讨提供了有价值的预测.
\end{abstract}

关键词＼cjkstart量子化学; 组合方法; 硫酸气溶胶; 永; 热力学函数

\section{A Combination Method of Quantum Chemistry and Its Application to the Study of the Effects of Mercury on the Formation of Sulfuric Acid Aerosol}

\author{
Chengqiao Li Yibo Wang* \\ (School of Chemistry and Chemical Engineering, Guizhou University, Key Laboratory of \\ High Performance Computational Chemistry, Guiyang 550025, China)
}

\begin{abstract}
Haze weather has been occurring frequently in recent years, and studies have shown that its formation mechanism is related to the nucleation of sulfuric acid aerosols. How mercury and its compounds affect the formation and growth of sulfuric acid aerosols is completely unclear in polluted areas where sulfur and mercury coexist. In order to explore this problem theoretically, we established a new quantum chemistry combination method. Millions of cluster structures are generated by using the Molclus Genmer module. The cluster geometries are preliminarily optimized by the GFN2-xTB method and sorted according to the Gibbs free energy changes of the cluster generation process. The top 100 optimized structures were re-optimized using the B3PW91-D3(BJ) method. In order to accurately calculate the binding energy of clusters, we established a small benchmark set based on the composition and structural characteristics of the clusters studied in the project, and used the $\mathrm{CCSD}(\mathrm{T}) / \mathrm{CBS}$ method to calculate the binding energy of each cluster model in the benchmark set. Using the binding energy as a standard, the best method selected from 42 DFT methods is $\omega$ B97M-D3(BJ). The binding energy calculated by B3PW91$\mathrm{D} 3(\mathrm{BJ})$ is corrected by the results of the $\omega \mathrm{B} 97 \mathrm{M}-\mathrm{D} 3(\mathrm{BJ})$ method to obtain an accurate thermodynamic function value. It is worth mentioning that the combined method was used to study a total of 2.4 million structures in 8 cluster models formed by mercury and its typical compounds, sulfuric acid and water. The results show that polar compounds of mercury such as $\mathrm{HgSO}_{4}$ and $\mathrm{HgO}$, together with $\mathrm{Hg}_{2}{ }^{2+}$ and $\mathrm{Hg}^{2+}$, can promote sulfuric acid aerosol formation, while $\mathrm{Hg}$ and non-polar $\mathrm{HgCl}_{2}$ and $\mathrm{Hg}_{2} \mathrm{Cl}_{2}$ have almost no effect on the nucleation of sulfuric acid aerosol. In addition, we found that the conclusion holds true for the temperature interval of the troposphere at standard atmospheric pressure. This conclusion provides valuable predictions for the studies on sulfuric acid aerosol nucleation mechanisms under the combined sulfur and mercury pollution environment.

Keywords quantum chemistry; combination method; sulfuric acid aerosols; mercury; thermodynamic function
\end{abstract}

\section{1 引言}

近年来频繁发生严重的雾䨪天气，但其形成机制还 不明确, 研究大气气溶胶成核和生长机制是一个重要课 题. 硫酸作为气溶胶成核重要的前体物已形成共识 ${ }^{[1]}$, 而在硫和录复合污染地区, 关于录及其化合物是否会参 与或驱动硫酸气溶胶形成尚不清楚.
采是环境中一直被人们重视的一种重金属污染物. 其在大气中以不同的化学形态存在 ${ }^{[2]}$, 包括气态单质录 $\left(\mathrm{Hg}^{0}\right)$, 二价录 $\left(\mathrm{Hg}^{2+}\right)$ 和少量亚录 $\left(\mathrm{Hg}_{2}{ }^{2+}\right)$. 经研究发现大 气中的录对颗粒物或雾䨪的形成有重要影响, 2016 年, Hong 等 ${ }^{[3]}$ 对大气录浓度监测发现雾䨪天气录的浓度高 于非雾皬天气，李亮等 ${ }^{[4]}$ 通过实验观测也发现大气录浓

*E-mail: ybw@gzu.edu.cn

Received April 12, 2021; published June 1, 2021.

Supporting information for this article is available free of charge via the Internet at http://sioc-journal.cn.

Project supported by the National Natural Science Foundation of China (No. 21767005) and Science and Technology Plan Project of Guizhou Province (No. 20175788).

项目受国家自然科学基金(No. 21767005)和贵州省科技计划项目(No. 20175788)资助. 
度与 $\mathrm{PM}_{2.5}$ 呈显著正相关.

硫也是大气环境重要的污染源之一, 硫燃烧后生成 二氧化硫并在大气中被氧化和水化后生成硫酸. 目前已 有不少关于硫酸与水分子生成团族的理论工作发表. 1961 年, Doyle ${ }^{[5]}$ 首次提出了 $\left(\mathrm{H}_{2} \mathrm{SO}_{4}\right)\left(\mathrm{H}_{2} \mathrm{O}\right)$ 成核的理论, 并发现气溶胶成核速率和硫酸的浓度相关. 后来 Jaecker 等[6]建立了 $\mathrm{H}_{2} \mathrm{SO}_{4}$ 和 $\mathrm{H}_{2} \mathrm{O}$ 团族的多种模型. $\mathrm{Su}-$ carrat 等 ${ }^{[7]}$ 研究了 $\left(\mathrm{H}_{2} \mathrm{SO}_{4}\right)\left(\mathrm{H}_{2} \mathrm{O}\right)_{1 \sim 4}$ 团簇模型, 指出对流层 中硫酸水合物是重要的存在形式之一. 近年, Elm 研究 组 ${ }^{8}$ 报道了 $\left(\mathrm{H}_{2} \mathrm{SO}_{4}\right)\left(\mathrm{H}_{2} \mathrm{O}\right)_{1 \sim 15}$ 团簇模型, 发现硫酸通过与 4 个水分子的直接相互作用达到 “饱和” ，其余水分子 形成氢键网络分布在硫酸周围.

在采、硫复合污染条件下, 关于录及其化合物对气 溶胶成核影响的实验观测已有报道. 2015 年, Humphries 等 ${ }^{[9]}$ 对南冰洋和南极海面上颗粒物的形成事件进行观测, 发现录浓度与 $\mathrm{CN}_{3 \sim 10}$ (直径为 $3 \sim 10 \mathrm{~nm}$ 的粒子)浓度成 正相关, 并提出可能是录驱动硫酸等气溶胶物质成核, 但未见相关的理论工作发表.

在大气气溶胶团簇的理论研究中, 构建符合实际环 境特征的团簇模型是模拟成败的关键, 大气中水的浓度 远远高于污染物, 本工作团簇模型至少需要数十个水分 子才能将硫酸和采及其化合物围住，随着水分子数增加, 团簇异构体数目显著增多, 对计算机性能和计算方法提 出了严峻挑战. 在前人发表的不少研究工作中, 团簇初 始结构优化不得不借助半经验方法如 MNDOPM5/PM6/PM7 等来完成, 但这些半经验方法在参数化 时, 偏重于 $\mathrm{H}, \mathrm{C}, \mathrm{N}, \mathrm{O}, \mathrm{F}$ 等有机元素, 对 $\mathrm{d}$ 轨道参与成 键的重元素考虑不充分, 计算结果不可靠, 特别是本工 作团簇模型中涉及不同价态的录, 不宜使用. 若采用密 度泛函及其色散校正方法完成数以百万计团簇的初始 几何结构优化, 远远超出目前计算机运算能力水平.

因此, 本工作发展了一套基于量子化学的组合计算 方法, 采用 Molclus Genmer 模块 ${ }^{[10]}$ 对每一种团簇生成大 量的初始构型, 首先使用 Grimme 等 ${ }^{[11]}$ 近年发展的自洽 紧束缚 GFN2-xTB 方法逐一优化初始结构. 用 Molclus Isostat 模块去除重复结构, 并以其生成过程 Gibbs 自由 能变大小排序, 以获得最可能自发形成的团簇序列. 为 了获得更加可靠的团族结构, 用密度泛函 B3PW91D3(BJ)方法再次优化每种团簇上述序列前 100 个结构、 计算热力学函数, 其中团簇生成过程中结合能是准确计 算 Gibbs 自由能变的关键, 我们根据本工作研究团簇模 型组成和结构特征建立了测试集, 计算并建立 $\operatorname{CCSD}(\mathrm{T}) / \mathrm{CBS}^{[12]}$ 结合能标准, 笁选出合适的密度泛函 及其色散校正方法, 以精确计算结合能. 并在计算过程 中十分慎重地处理了基组重叠误差(basis set superposition error, BSSE) 问题. 最后, 通过分析团簇生成过程的 热力学函数变化, 判断不同类型的录及其化合物对硫酸
气溶胶成核的影响，期望为相关气溶胶成核机制的实验 研究提供有价值的预测.

\section{2 组合计算方法及实施过程}

\section{1 团簇几何结构}

团簇模型构建原则. 大气气溶胶在临界核成核阶段, 形成直径 $1 \sim 3 \mathrm{~nm}$ 尺度分子团族的过程是成核的关键步 骤, 建立符合研究问题特征的团簇模型十分重要 ${ }^{[13]}$. 前 人已发表的不少大气团族计算工作中，团族模型采用多 个硫酸与多个有机酸或有机胺构成, 夸大了污染物的浓 度, 更重要的是忽视了大气环境中水的存在及其重要作 用 ${ }^{[14]}$, 建模的思路值得商榷.

我们在建立团簇模型过程中, 考虑到即使在录、硫 复合污染地区, 大气中录和硫也是痕量的, 而水分子在 大气中主要以团族分子形式存在 ${ }^{[15]}$, 且含量较高, 同时 具有较强的形成氢键能力，从气相到团簇、再到气溶胶 的成核过程，水的作用完全不可忽视. 所以构建的模型 采用 1 个硫酸、 1 个录或化合物，加上 30 个水，使录和 硫酸被水分子包围, 这较为符合大气环境的实际情况和 各种成分含量的比例, 其中根据大气环境中采的存在形 态，包括单质采、离子录和一价录、二价录的极性或非 极性化合物, 建立了 $\left(\mathrm{H}_{2} \mathrm{SO}_{4}\right)\left(\mathrm{H}_{2} \mathrm{O}\right)_{30},(\mathrm{Hg})\left(\mathrm{H}_{2} \mathrm{SO}_{4}\right)-$ $\left(\mathrm{H}_{2} \mathrm{O}\right)_{30},\left(\mathrm{HgCl}_{2}\right)\left(\mathrm{H}_{2} \mathrm{SO}_{4}\right)\left(\mathrm{H}_{2} \mathrm{O}\right)_{30},\left(\mathrm{Hg}_{2} \mathrm{Cl}_{2}\right)\left(\mathrm{H}_{2} \mathrm{SO}_{4}\right)\left(\mathrm{H}_{2} \mathrm{O}\right)_{30}$, $(\mathrm{HgO})\left(\mathrm{H}_{2} \mathrm{SO}_{4}\right)\left(\mathrm{H}_{2} \mathrm{O}\right)_{30},\left(\mathrm{HgSO}_{4}\right)\left(\mathrm{H}_{2} \mathrm{SO}_{4}\right)\left(\mathrm{H}_{2} \mathrm{O}\right)_{30}$ 和 $\left(\mathrm{Hg}_{2}{ }^{2+}\right)-$ $\left(\mathrm{H}_{2} \mathrm{SO}_{4}\right)\left(\mathrm{H}_{2} \mathrm{O}\right)_{30},\left(\mathrm{Hg}^{2+}\right)\left(\mathrm{H}_{2} \mathrm{SO}_{4}\right)\left(\mathrm{H}_{2} \mathrm{O}\right)_{30}$ 共 8 种团簇模型.

初始团簇结构生成. 本工作首先使用 Molclus Genmer 模块随机生成 8 种团簇的初始结构, 每种体系 30 万, 共 240 万. 在此过程中设置每一步移动单体的距离 step 为 0.01 , 控制加入单体的顺序参数 ishuffle 为 1 , 设定单 体的中心与体系中心最远距离 $\operatorname{rmax}$ 为 8 , 即团簇最大半 径为 $0.8 \mathrm{~nm}$, 其它参数均为采用默认值, 避免漏掉团簇 生成过程 Gibbs 自由能变大的结构. 目前该方法已成功 地应用于团簇结构的相关研究 ${ }^{[16-17]}$.

团簇几何结构预优化. 本工作建立的含录团簇包含 30 多个分子，原子数在 100 左右, 初始团簇共 240 万个. 为了获得团簇生成过程 Gibbs 自由能变极大的几何结构 序列, 我们使用 Molclus 程序调用 xTB 程序, 对所有初 始结构逐一进行几何构型优化和振动分析.

GFN2-xTB 是一种精确、广义参数化的基于静电多 极展开和依赖电子密度色散贡献的自洽紧束缚量子化 学新方法, 主要用于快速计算分子能量、几何构型、振 动频率及非共价相互作用能, 在团簇结构快速优化 ${ }^{[18]}$, 含过渡金属体系的模拟计算中获得成功 ${ }^{[19]}$.

GFN2-xTB 通过累积原子多极矩的短程阻尼相互作 用来处理各向异性的二阶密度波动效应, 其能量成分与 $\delta \rho$ 的不同阶次关联，总能量表达为:

$$
E_{\mathrm{GFN} 2-\mathrm{xTB}}=E_{\mathrm{rep}}+E_{\mathrm{disp}}+E_{\mathrm{EHT}}+E_{\mathrm{IES}+\mathrm{IXC}}
$$




$$
+E_{\mathrm{AES}}+E_{\mathrm{AXC}}+G_{\mathrm{Fermi}}
$$

$E_{\mathrm{rep}}$ 为经典排斥能，该项表达为原子对势能:

$$
E_{\text {rep }}=\sum_{A B} \frac{Y_{A}^{\text {eff }} Y_{B}^{\text {eff }}}{R_{A B}} e^{-\left(\alpha_{A} \alpha_{B}\right)^{\frac{1}{2}}}\left(R_{A B}\right)^{k_{\text {rep }}}
$$

其中 $Y_{A}^{\text {eff }}$ 和 $Y_{B}^{\text {eff }}$ 定义了排斥强度, 连同 $\alpha_{A}$ 和 $\alpha_{B}$, 是依赖 元素的参数. 对于参数 $k_{\mathrm{rep}}$, 如果两个原子均为 $\mathrm{H}$ 或 $\mathrm{He}$, 则取 1, 否则取 3/2. $E_{\mathrm{EHT}}$ 表示扩展 Hückel 方法的能量 贡献，描述紧束缚方法中的共价键成分，表达为:

$$
E_{\mathrm{EHT}}=\sum_{i} n_{i}\left\langle\Psi_{i}\left|\widehat{H}_{0}\right| \Psi_{i}\right\rangle \equiv \sum_{\kappa} \sum_{\lambda} P_{\kappa \lambda} H_{\lambda \kappa}
$$

$E_{\text {disp }}$ 为密度依赖色散能, 采用 DFT-D4 色散模型通过自 洽变分获得, 可有效地将电子结构影响纳入色散能. $E_{\mathrm{IES}+\mathrm{IXC}}$ 表示各向同性静电和交换相关能量, $E_{\mathrm{AES}+\mathrm{AXC}}$ 表示各向异性静电和各向异性交换相关能量, $G_{\mathrm{Fermi}}$ 与 有限温度下, 电子自由能熵贡献有关 ${ }^{[1]}$.

能量表达式(1)采用自洽场方法求解. 总能量的 Hamilton 矩阵元分解为:

$$
F_{\kappa \lambda}=H_{\kappa \lambda}+F_{\kappa \lambda}^{\mathrm{IES}+\mathrm{IEC}}+F_{\kappa \lambda}^{\mathrm{AEC}}+F_{\kappa \lambda}^{\mathrm{AXC}}+F_{\kappa \lambda}^{\mathrm{D} 4}
$$

与 Hartree-Fock 类似, 此矩阵称为 TB-Fock 矩阵.

此外, GFN2-xTB 方法包含全局性参数和依赖元素 性参数, 后者已对元素周期表中前 86 号元素进行了参 数化. 该方法具有改进的静电贡献表达和更一致性的参 数化, 较其它半经验方法能够更好地描述体系的电子密 度.

团簇几何结构去重和排序. 每种团簇从 30 万个结 构出发, 用 GFN2-xTB 方法优化得到结构中, 包含了大 量重复结构, 需要从团簇几何结构相似性和能量因素归 类并排除重复结构. 能量因素通常有两种标准, 一种是 按照体系总能量去重、排序 ${ }^{[20]}$, 获得能量高低团簇系列; 另一种是按团簇生成过程 Gibbs 自由能变去重、排序, 它能够更好地预测团簇自发形成的可能性因素. 本工作 选择第二种方法, 使用了 Molclus Isostat 模块进行团簇 几何结构相似性和 Gibbs 自由能变去重、排序, 相似性 判断阈值取 0.25 , 结果见支持信息 $(\mathrm{SI})$ 表 S12. 每种团簇 保留前 100 个结构作为下一步 DFT 优化的初始结构.

团簇几何结构再优化. B3PW91 是由 Becke 的三参 数混合交换泛函(B3)和 Perdew, Wang(PW91)的相关泛函 组成的混合交换相关泛函 ${ }^{[21-22]}$. Amaro-Estrada 等以 $(\mathrm{HgClOH})\left(\mathrm{H}_{2} \mathrm{O}\right)_{n}$ 体系 $(n=0,1$ 和 2)为研究对象, 使用 MP2/aug-cc-pVTZ 计算结果为参考, 在相同基组下测试 了多种 DFT 方法, 其中 B3PW91 加上 D3(BJ)色散校正 获得的几何结构参数、振动频率和结合能较接近 MP2 结 果, 被认为用于录和水团簇热力学贡献计算是较合适 的 ${ }^{[23-24]}$.

因此, 本工作对每种团簇经去重、按 Gibbs 自由能 变大小排序的前 100 个结构, 用 B3PW91-D3(BJ)方法重
新优化并计算其振动频率，结合能经过校正后，再次去 重并按 Gibbs 自由能变排序, 得到最可能自发形成的团 簇结构序列.

\section{2 团簇结合能与热力学函数计算}

使用量子化学方法计算团簇生成过程的 Gibbs 自由 能变 $\Delta G_{\text {bind }}$ 表达为:

$$
\Delta G_{\text {bind }}=\Delta E_{\text {bind }}+\Delta G_{\text {thermal }}
$$

式中 $\Delta E_{\text {bind }}$ 表示团簇的结合能, $\Delta G_{\text {thermal }}$ 表示 Gibbs 自 由能变的热力学贡献 ${ }^{[14]}$.

$$
\begin{aligned}
\Delta E_{\text {bind }} & =E_{\text {cluster }}-\sum_{i} E_{\text {monomers }, i} \\
\Delta G_{\text {bind }} & =G_{\text {cluster }}-\sum_{i} G_{\text {monomers }, i}
\end{aligned}
$$

$E_{\text {cluster }}$ 和 $G_{\text {cluster }}$ 为团簇的总能量和总 Gibbs 自由能, $E_{\text {monomers }, i}$ 为自由单体的能量和 Gibbs 自由能.

计算实践表明 $\Delta E_{\text {bind }}$ 数值在 $\Delta G_{\text {bind }}$ 占比高, 其计算 精度对 $\Delta G_{\text {bind }}$ 可靠性影响大, 精确计算结合能十分重要, B3PW91-D3(BJ)/def2-SV(P) 方法获得结合能还不够精 确.

为了寻找适于本工作所研究团簇结合能的高精度 密度泛函及其色散校正方法. 我们从本工作建立的团簇 模型中, 通过减少水分子数量, 简化出 $\left(\mathrm{H}_{2} \mathrm{SO}_{4}\right)\left(\mathrm{H}_{2} \mathrm{O}\right)_{2}$, $(\mathrm{Hg})\left(\mathrm{H}_{2} \mathrm{O}\right)_{2}, \quad\left(\mathrm{HgCl}_{2}\right)\left(\mathrm{H}_{2} \mathrm{O}\right)_{2}, \quad(\mathrm{HgO})\left(\mathrm{H}_{2} \mathrm{O}\right)_{2}, \quad(\mathrm{Hg})\left(\mathrm{H}_{2} \mathrm{SO}_{4}\right)$ $\left(\mathrm{H}_{2} \mathrm{O}\right),(\mathrm{Hg})\left(\mathrm{H}_{2} \mathrm{SO}_{4}\right)\left(\mathrm{H}_{2} \mathrm{O}\right)_{2},(\mathrm{Hg})\left(\mathrm{H}_{2} \mathrm{SO}_{4}\right)\left(\mathrm{H}_{2} \mathrm{O}\right)_{3},\left(\mathrm{HgCl}_{2}\right)-$ $\left(\mathrm{H}_{2} \mathrm{SO}_{4}\right)\left(\mathrm{H}_{2} \mathrm{O}\right),\left(\mathrm{HgCl}_{2}\right)\left(\mathrm{H}_{2} \mathrm{SO}_{4}\right)\left(\mathrm{H}_{2} \mathrm{O}\right)_{2}$ 和 $\left(\mathrm{HgCl}_{2}\right)\left(\mathrm{H}_{2} \mathrm{SO}_{4}\right)-$ $\left(\mathrm{H}_{2} \mathrm{O}\right)_{3}$ 共 10 个小团簇, 用 MP2/aug-cc-pVTZ 方法 ${ }^{[25]}$ 逐 一优化出几何结构(见 SI 图 S1 和表 S1), 建立了测试集, 并用达到化学精度的 $\operatorname{CCSD}(\mathrm{T}) / \mathrm{CBS}$ 方法 ${ }^{[12]}$ 计算出结合 能标准值.

$\operatorname{CCSD}(\mathrm{T}) / \mathrm{CBS}$ 方法表达为:

$$
E_{\mathrm{CBS}}^{\mathrm{CCSD}(\mathrm{T})}=E_{\mathrm{CBS}}^{\mathrm{MP2}}+\left(E_{\text {aug-cc-pVTZ }}^{\mathrm{CCSD}(\mathrm{T})}-E_{\text {aug-cc-pVTZ }}^{\mathrm{MP2}}\right)
$$

$E_{\mathrm{CBS}}^{\mathrm{MP} 2}$ 表示在完备基组水平下 $\mathrm{MP} 2$ 的结合能，其表达为:

$$
\begin{aligned}
E_{\mathrm{CBS}}^{\mathrm{MP} 2} & =E_{\mathrm{SCF}}^{\infty}+E_{\mathrm{corr}}^{\mathrm{MP} 2, \infty} \\
E_{\mathrm{corr}}^{\mathrm{MP} 2, \infty} & =\frac{X^{\beta} E_{\mathrm{corr}}^{X}-Y^{\beta} E_{\mathrm{corr}}^{Y}}{X^{\beta}-Y^{\beta}}
\end{aligned}
$$

式(9)中 $E_{S C F}^{\infty}$ Hartree-Fock 极限总能量用 aug-cc-pVQZ 基 组计算值. MP $2 / C B S$ 相关能按式(10)两点外推, 使用 aug-cc-pVTZ 和 aug-cc-pVQZ 基组时 $X$ 和 $Y$ 分别取 3 和 $4, \beta$ 取 $3^{[26]}$. CCSD(T) 高级项校正用 aug-cc-pVTZ 基组以 保证其精度.

由于外推至完备基极限的 $\operatorname{CCSD}(\mathrm{T}) / \mathrm{CBS}$ 结合能的 BSSE 非常小，已忽略，不再考虑. 计算结果见 SI 表 S2.

用测试集 $\operatorname{CCSD}(\mathrm{T}) / \mathrm{CBS}$ 结合能为标准, 从研究非 共价相互作用的 B3LYP, PBE0 及色散校正, $\omega$ B97X-V, M06-2X, B972-PFD ${ }^{[27]}$ 等 42 种密度泛函方法中, 用 def2QZVPP 基组, 篮选出 $\omega B$ 97M-D3(BJ)是适合于本工作团 簇计算的最优性价比方法，其 RMSD 误差最小为 2.09 
$\mathrm{kJ} / \mathrm{mol}, \mathrm{MAD}, \mathrm{MD}, \mathrm{MAX}$ 误差分别为 $0.21,1.67,4.31$ $\mathrm{kJ} / \mathrm{mol}$, 达到 $4.18 \mathrm{~kJ} / \mathrm{mol}$ 化学精度范围, 计算结果见图 1 和 SI 表 S3.

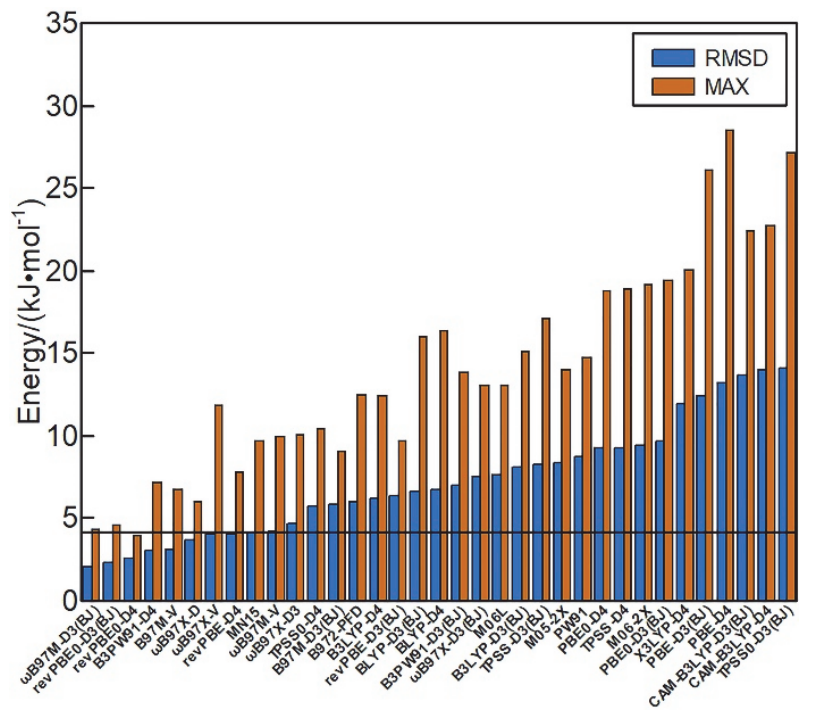

图 1 DFT/def2-QZVPP 计算测试集的 RMSD 和 MAX 比较, MAX 取 绝对值

Figure 1 Comparison of RMSD and MAX of DFT/def2-QZVPP calculation benchmark set, MAX takes the absolute value

$\omega B$ 97M-D3(BJ)方法是 Najibi 和 Goerigk 将 $\omega$ B97M$\mathrm{V}$ 方法中的 VV10 非局域校正替换成了 D3(BJ) 色散校 正, 构成的一种新的密度泛函色散校正方法 ${ }^{[28]}$. 对于本 工作含录、硫酸及水的氢键团簇, $\omega$ B $97 \mathrm{M}-\mathrm{D} 3(\mathrm{BJ})$ 优于 $\omega \mathrm{B} 97 \mathrm{M}-\mathrm{V}$ 方法出人预料.

据此, 本工作使用 $\omega B$ B97M-D3(BJ)/def2-QZVPP 方 法计算的结合能替代 B3PW91-D3(BJ)/def2-SV(P)结合 能, 式(5)表达为组合形式:

$$
\Delta G_{\text {bind }}=\Delta E_{\text {bind }}^{\omega \mathrm{B} 97 \mathrm{M}-\mathrm{D} 3(\mathrm{BJ})}+\Delta G_{\text {thermal }}^{\mathrm{B} 3 \mathrm{PW} 91-\mathrm{D} 3(\mathrm{BJ})}
$$

$\Delta E_{\mathrm{bind}}^{\omega \mathrm{B} 97 \mathrm{M}-\mathrm{D} 3(\mathrm{BJ})}$ 是 $\omega \mathrm{B} 97 \mathrm{M}-\mathrm{D} 3(\mathrm{BJ})$ 计算的结合能.

$\Delta G_{\text {thermal }}^{\text {B3PW91-D3(BJ) }}$ 是 B3PW91-D3(BJ)计算的 Gibbs 自 由能变的热力学贡献:

$$
\begin{gathered}
\Delta G_{\text {thermal }}^{\mathrm{B} 3 \mathrm{PW} 1-\mathrm{D} 3(\mathrm{BJ})}=\Delta G_{\text {bind }}^{\mathrm{B} 3 \mathrm{PW} 91-\mathrm{D} 3(\mathrm{BJ})} \\
-\Delta E_{\text {bind }}^{\mathrm{B3PW} 91-\mathrm{D} 3(\mathrm{BJ})}
\end{gathered}
$$

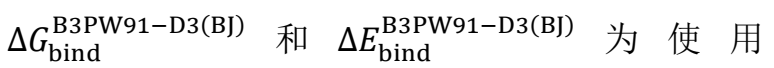
B3PW91-D3(BJ)计算的 Gibbs 自由能变和结合能.

结合能计算的 BSSE 校正. 在结合计算过程中, 我 们十分慎重地分类处理了基函数的 BSSE 误差问题: 第 一, $\omega$ B97M-D3(BJ)/def2-QZVPP 计算部分, 由于使用了 4-Zeta 级别的 def2-QZVPP 基组, 已接近完备基, 根据 Head-Gordon 组在对同系列密度泛函基函数效应的研究 结论, 可不做 BSSE 校正 ${ }^{[29-30]}$; 第二, 式(12)中减去的 B3PW91-D3(BJ)/def2-SV(P)结合能, 由于 $\operatorname{def} 2-S V(P)$ 基 组过小, 若忽略 BSSE, 将会污染 Gibbs 自由能变的计算 结果.
对于大团簇的 BSSE 校正，同时要考虑生成团簇后 单体分子形变能, 传统 CP (CounterPoise)校正方法 ${ }^{[31]}$ 难 以实施. 因此前人已发表的不少工作中, 在计算团簇 Gibbs 自由能变时, 通常放弃了小基组下获得结合能的 BSSE 校正, 导致 $\Delta G$ 计算结果不够准确.

我们采用了 Grimme 研究组提出的 gCP (geometrical CounterPoise) 校正方法 ${ }^{[32]}$ 完成了 $\operatorname{def} 2-\mathrm{SV}(\mathrm{P})$ 基组的 $\mathrm{BSSE}$ 校正, 以避免 $\Delta G_{\mathrm{thermal}}^{\mathrm{B} 3 \mathrm{PW} 91-\mathrm{D} 3(\mathrm{BJ})}$ 的数值被污染. 本 工作所研究的 8 种团簇在使用 B3PW91-D3(BJ)/def2$\mathrm{SV}(\mathrm{P})$ 计算时, 其 BSSE 均大于 $900 \mathrm{~kJ} / \mathrm{mol}$, 计算结果见 SI 表 S13.

$\mathrm{gCP}$ 校正对分子体系能量添加校正 $E_{\mathrm{gCP}}$ 项:

$$
E_{\mathrm{gCP}}=\sigma \cdot \sum_{a}^{N} \sum_{b \neq a}^{N} e_{a}^{\mathrm{miss}} \cdot f_{\mathrm{dec}}\left(R_{a b}\right)
$$

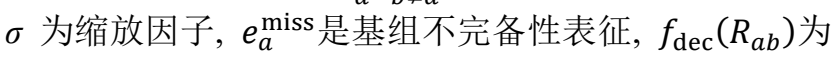
衰减函数.

\section{3 计算过程}

本工作建立的量子化学组合计算方法流程如图 2 所 示. 在计算过程中, 用 Molclus 程序 ${ }^{[10]}$ 生成团族的初始 结构和去重、排序，用 xTB 程序 ${ }^{[33]}$ 的 GFN2-xTB 方法对 初始结构预优化和振动频率计算. 用 Gaussian16 程序 ${ }^{[34]}$ 完成 B3PW91-D3(BJ)等 DFT 的单点能、结构优化和热 力学函数计算等, 用 ORCA 4.2.1 程序[35]完成 $\omega \mathrm{B} 97 \mathrm{M}-$ D3(BJ)等 DFT 计算和 gCP BSSE 校正, 用 Molpro 2018.2 程序[36]完成 $\operatorname{CCSD}(\mathrm{T}) / \mathrm{CBS}$ 计算. 全部计算工作在贵州 省高性能计算化学重点实验室 GHPCC 的 48 节点并行 计算系统上完成, 历时约一年半时间.

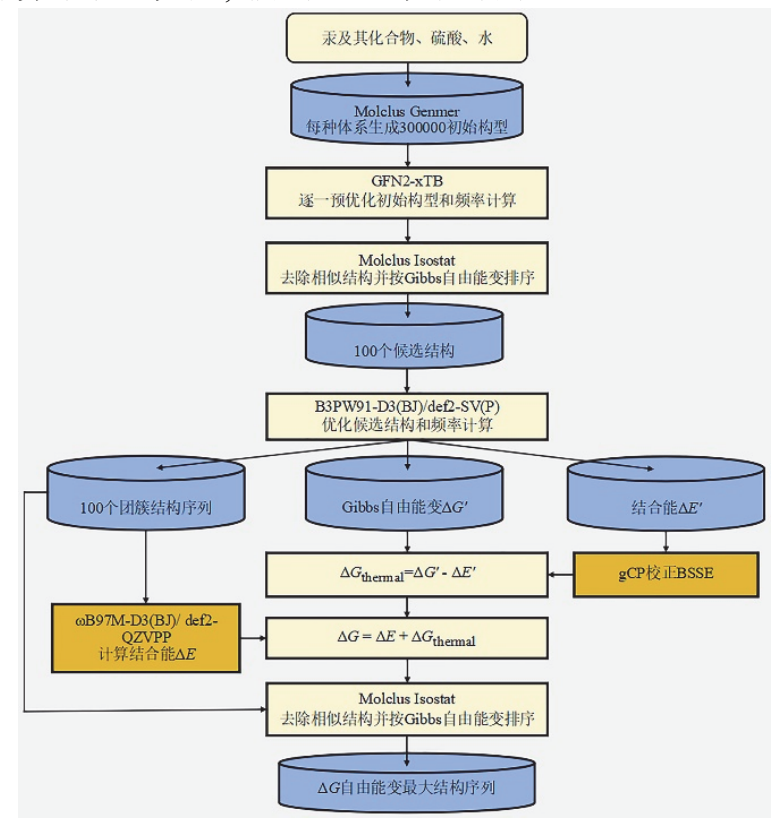

图 2 组合方法的计算流程示意图

Figure 2 Schematic representation of the calculation process of the combined method

$\Delta G^{\prime}$ represent $\Delta G_{\text {bind }}^{\text {B3P1-D3(BJ) }}, \Delta E^{\prime}$ represent $\Delta E_{\text {bind }}^{\mathrm{B3PW} 91-\mathrm{D} 3(\mathrm{BJ})}, \Delta E$ represent $\Delta E_{\text {bind }}^{\omega \mathrm{Bg} \text { M }-\mathrm{D} 3(\mathrm{BJ})}$ 


\section{3 结果与讨论}

使用本工作所建立的量子化学的组合方法, 获得了 寽及其化合物、硫酸和水生成的 8 种团簇的稳定序列及 其对应结合能和热力学函数. 图 3 为在 $298.15 \mathrm{~K}$ 和 0.1 $\mathrm{MPa}$ 标准态下, 8 种团簇生成过程 Gibbs 自由能变最大 (绝对值)的结构, 表 1 给出了结合能和 Gibbs 自由能变 $(\Delta G)$ 、焓变 $(\Delta H)$ 和熵变 $(\Delta S)$ 等热力学函数. 其中 average 和 $\max$ 分别表示按 $\Delta G$ 从大到小排序后前 5 个结构的平 均值和最大值, 详细计算结果见 SI 表 $\mathrm{S} 4 \sim \mathrm{S} 11$.

\section{1 团簇的结合能与稳定性分析}

通过 B3PW91-D3(BJ)/def2-SV(P) 优化后的结构都 不存在虚频, 团簇直径在 $1.5 \mathrm{~nm}$ 左右. 通过观察图 3 结
构发现，在微水环境下，团簇中的分子发生部分电离以 水合离子形式存在，这与文献结论保持一致 ${ }^{[37]}$.

通过表 1 中 $\Delta E$ 的数据可以发现: 其一, 含有 $\mathrm{Hg}$, $\mathrm{Hg}_{2} \mathrm{Cl}_{2}$ 和 $\mathrm{HgCl}_{2}$ 参与生成团簇的过程(2), (3)和(6), 其 $\Delta E$ 的平均值和最大值与过程(1)变化较小, 过程(6)的 $\Delta E$ 平 均值(绝对值, 以下同)较过程(1)仅增大 $20.16 \mathrm{~kJ} / \mathrm{mol}$, 说 明 $\mathrm{Hg}, \mathrm{Hg}_{2} \mathrm{Cl}_{2}$ 和 $\mathrm{HgCl}_{2}$ 对团簇稳定性的影响很小. 另外 从几何结构也可看出, $\mathrm{Hg}$ 处于团簇边缘, $\mathrm{Hg}_{2} \mathrm{Cl}_{2}$ 和 $\mathrm{HgCl}_{2}$ 周围形成空腔, 不利于团簇稳定. 其二, 包含 $\mathrm{HgSO}_{4}$ 和 $\mathrm{HgO}$ 的过程(4)和(5)与过程(1)相比, $\Delta E$ 明显变大, 过程 (4)和过程(5) $\Delta E$ 的平均值分别增大 $493.25,410.62$ $\mathrm{kJ} / \mathrm{mol}$, 则说明 $\mathrm{HgSO}_{4}$ 和 $\mathrm{HgO}$ 使硫酸气溶胶的稳定性 增强. 其三, 对于含录离子的体系, 与过程(1)相比,

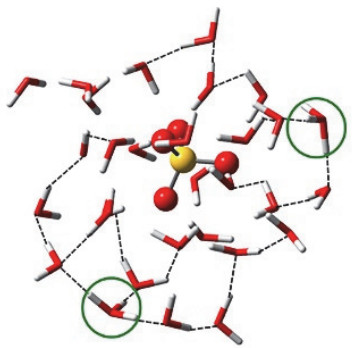

$\left(\mathrm{H}_{2} \mathrm{SO}_{4}\right)\left(\mathrm{H}_{2} \mathrm{O}\right)_{30}$

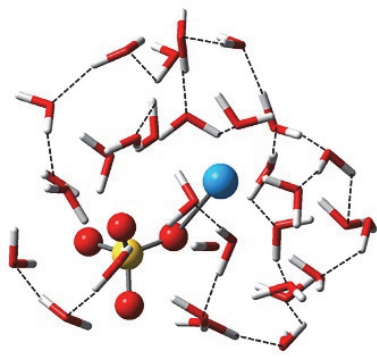

$(\mathrm{HgO})\left(\mathrm{H}_{2} \mathrm{SO}_{4}\right)\left(\mathrm{H}_{2} \mathrm{O}\right)_{30}$

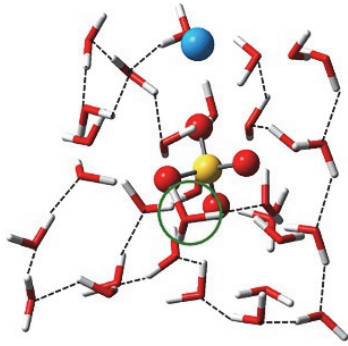

$(\mathrm{Hg})\left(\mathrm{H}_{2} \mathrm{SO}_{4}\right)\left(\mathrm{H}_{2} \mathrm{O}\right)_{30}$

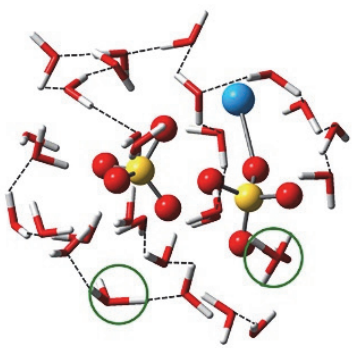

$\left(\mathrm{HgSO}_{4}\right)\left(\mathrm{H}_{2} \mathrm{SO}_{4}\right)\left(\mathrm{H}_{2} \mathrm{O}\right)_{30}$

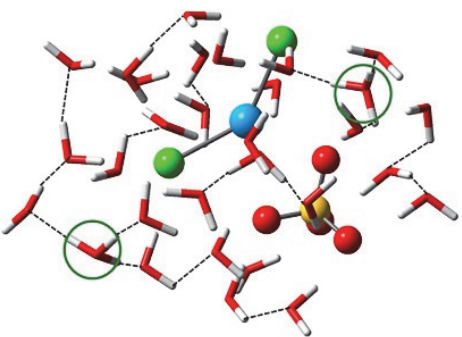

$\left(\mathrm{HgCl}_{2}\right)\left(\mathrm{H}_{2} \mathrm{SO}_{4}\right)\left(\mathrm{H}_{2} \mathrm{O}\right)_{30}$

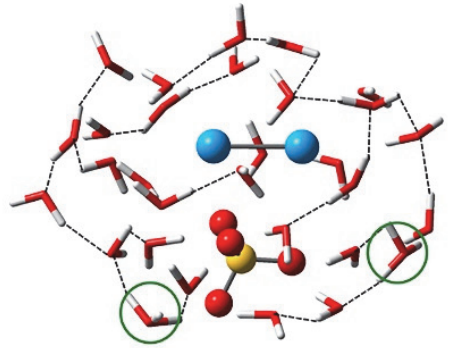

$\left(\mathrm{Hg}_{2}{ }^{2+}\right)\left(\mathrm{H}_{2} \mathrm{SO}_{4}\right)\left(\mathrm{H}_{2} \mathrm{O}\right)_{30}$

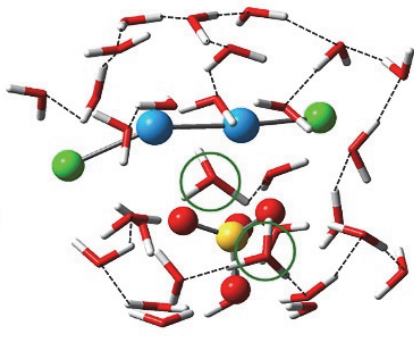

$\left(\mathrm{Hg}_{2} \mathrm{Cl}_{2}\right)\left(\mathrm{H}_{2} \mathrm{SO}_{4}\right)\left(\mathrm{H}_{2} \mathrm{O}\right)_{30}$

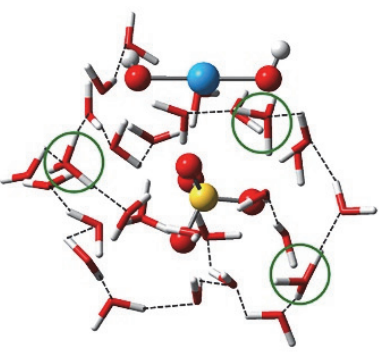

$\left(\mathrm{Hg}^{2+}\right)\left(\mathrm{H}_{2} \mathrm{SO}_{4}\right)\left(\mathrm{H}_{2} \mathrm{O}\right)_{30}$

图 3 B3PW91-D3(BJ)/def2-SV(P)方法计算 8 种团簇生成过程的 Gibbs 自由能变最大结构

Figure 3 The Gibbs free energy change maximum structure of the 8 cluster formation processes calculated at the B3PW91-D3(BJ)/def2-SV(P) level of theory

表 18 种团簇生成过程的结合能和热力学函数(单位: $\mathrm{kJ} / \mathrm{mol}$ )

Table 1 Binding energies and thermodynamic functions of 8 cluster formation processes (unit: $\mathrm{kJ} / \mathrm{mol}$ )

\begin{tabular}{|c|c|c|c|c|c|c|c|c|c|}
\hline \multirow{2}{*}{ No. } & \multirow{2}{*}{ Reaction } & \multicolumn{2}{|c|}{$\Delta E$} & \multicolumn{2}{|c|}{$\Delta H$} & \multicolumn{2}{|c|}{$\Delta S$} & \multicolumn{2}{|c|}{$\Delta G$} \\
\hline & & average & $\max$ & average & $\max$ & average & $\max$ & average & $\max$ \\
\hline 1 & $\mathrm{H}_{2} \mathrm{SO}_{4}+30 \mathrm{H}_{2} \mathrm{O}=>\left(\mathrm{H}_{2} \mathrm{SO}_{4}\right)\left(\mathrm{H}_{2} \mathrm{O}\right)_{30}$ & -1391.81 & -1426.16 & -2367.10 & -2388 & -4.52 & -4.56 & -809.65 & -81 \\
\hline 2 & $\mathrm{Hg}+\mathrm{H}_{2} \mathrm{SO}_{4}+30 \mathrm{H}_{2} \mathrm{O}=>(\mathrm{Hg})\left(\mathrm{H}_{2} \mathrm{SO}_{4}\right)\left(\mathrm{H}_{2} \mathrm{O}\right)_{30}$ & -1417.62 & -1430.68 & -2397.68 & -2434.67 & -4.60 & -4.64 & -791.74 & -799.19 \\
\hline 3 & $\mathrm{Hg}_{2} \mathrm{Cl}_{2}+\mathrm{H}_{2} \mathrm{SO}_{4}+30 \mathrm{H}_{2} \mathrm{O}=>\left(\mathrm{Hg}_{2} \mathrm{Cl}_{2}\right)\left(\mathrm{H}_{2} \mathrm{SO}_{4}\right)\left(\mathrm{H}_{2} \mathrm{O}\right)_{30}$ & -1429.97 & -1468.29 & -2512.99 & -2550.94 & -4.64 & -4.69 & -749.61 & -782.03 \\
\hline 4 & $\mathrm{HgSO}_{4}+\mathrm{H}_{2} \mathrm{SO}_{4}+30 \mathrm{H}_{2} \mathrm{O}=>\left(\mathrm{HgSO}_{4}\right)\left(\mathrm{H}_{2} \mathrm{SO}_{4}\right)\left(\mathrm{H}_{2} \mathrm{O}\right)_{30}$ & -1885.06 & -1896.52 & -2857.13 & -2888.97 & -4.73 & -4.77 & -1291.35 & -1297.54 \\
\hline 5 & $\mathrm{HgO}+\mathrm{H}_{2} \mathrm{SO}_{4}+30 \mathrm{H}_{2} \mathrm{O}=>(\mathrm{HgO})\left(\mathrm{H}_{2} \mathrm{SO}_{4}\right)\left(\mathrm{H}_{2} \mathrm{O}\right)_{30}$ & -1802.43 & -1826.57 & -2787.05 & -2833.99 & -4.69 & -4.73 & -1177.92 & -1186.08 \\
\hline 6 & $\mathrm{HgCl}_{2}+\mathrm{H}_{2} \mathrm{SO}_{4}+30 \mathrm{H}_{2} \mathrm{O}=>\left(\mathrm{HgCl}_{2}\right)\left(\mathrm{H}_{2} \mathrm{SO}_{4}\right)\left(\mathrm{H}_{2} \mathrm{O}\right)_{30}$ & -1411.97 & -1420.72 & -2461.15 & -2486.97 & -4.64 & -4.69 & -766.89 & -774.04 \\
\hline 7 & $\mathrm{Hg}_{2}{ }^{2+}+\mathrm{H}_{2} \mathrm{SO}_{4}+30 \mathrm{H}_{2} \mathrm{O}=>\left(\mathrm{Hg}_{2}{ }^{2+}\right)\left(\mathrm{H}_{2} \mathrm{SO}_{4}\right)\left(\mathrm{H}_{2} \mathrm{O}\right)_{30}$ & -2271.91 & -2295.89 & -3413.64 & -3456.49 & -4.69 & -4.73 & -1695.44 & -1700.54 \\
\hline 8 & $\mathrm{Hg}^{2+}+\mathrm{H}_{2} \mathrm{SO}_{4}+30 \mathrm{H}_{2} \mathrm{O}=>\left(\mathrm{Hg}^{2+}\right)\left(\mathrm{H}_{2} \mathrm{SO}_{4}\right)\left(\mathrm{H}_{2} \mathrm{O}\right)_{30}$ & -2715.04 & -2749.18 & -3818.61 & -3849.03 & -4.60 & -4.69 & -2051.08 & -2069.20 \\
\hline
\end{tabular}


$\mathrm{Hg}_{2}{ }^{2+}$ 和 $\mathrm{Hg}^{2+}$ 参与生成团簇过程(7)和过程(8)的结合能 明显更大, 过程(8)结合能增大了近两倍, 表明 $\mathrm{Hg}_{2}{ }^{2+}$ 和 $\mathrm{Hg}^{2+}$ 参与生成团簇, 能显著增强团簇的稳定性.

\section{2 团簇的热力学函数分析}

通过表 1 中焓变 $\Delta H$ 数据(绝对值, 以下同)可以发 现, 所有团簇生成 $\Delta H$ 数值均较大, 过程(8)平均值达到 $3849.03 \mathrm{~kJ} / \mathrm{mol}$, 表明生成团簇的过程中放出大量的热. 将过程(2 8) 与过程(1)相比较发现, 当 $\mathrm{Hg}, \mathrm{Hg}_{2} \mathrm{Cl}_{2}$ 和 $\mathrm{HgCl}_{2}$ 参与生成团簇时, $\Delta H$ 变化较小, 过程(2) $\Delta H$ 的平 均值仅比过程(1)增加 $30.58 \mathrm{~kJ} / \mathrm{mol}$. 而 $\mathrm{HgSO}_{4}$ 和 $\mathrm{HgO}$ 参 与生成团簇过程(4)和(5), 其 $\Delta H$ 明显大于过程(1), 过程 (4) $\Delta H$ 的最大值达 $2888.97 \mathrm{~kJ} / \mathrm{mol}$, 比过程(1)增大 $500.66 \mathrm{~kJ} / \mathrm{mol}$. 说明 $\mathrm{HgSO}_{4}, \mathrm{HgO}$ 和采离子参与生成团 簇时, 能够放出更多的热.

分析表 1 中 Gibbs 自由能变 $\Delta G$ 数据发现, 所有团 簇在 $298.15 \mathrm{~K}, 0.1 \mathrm{MPa}$ 条件下都能够自发形成. 但将过 程(2 8)与过程(1)对比发现, 含有 $\mathrm{Hg}, \mathrm{Hg}_{2} \mathrm{Cl}_{2}$ 和 $\mathrm{HgCl}_{2}$ 参与生成团簇的过程(2), (3)和(6), 其 $\Delta G$ 平均值(绝对值, 以下同)均减小, 过程(3)的 $\Delta G$ 平均值减小 $60.04 \mathrm{~kJ} / \mathrm{mol}$, 这说明 $\mathrm{Hg}, \mathrm{Hg}_{2} \mathrm{Cl}_{2}$ 和 $\mathrm{HgCl}_{2}$ 降低了硫酸气溶胶自发形成 的可能性. 而 $\mathrm{HgSO}_{4}, \mathrm{HgO}$ 以及采离子参与生成团簇的 过程 $(4,5)$ 和 $(7,8)$, 这些过程均能明显地增大团簇生成 过程的 $\Delta G$, 特别是过程(8), 其 $\Delta G$ 最大值增大 1252.82 $\mathrm{kJ} / \mathrm{mol}$, 过程(4)和(5)的 $\Delta G$ 平均值也分别增大 481.70 和 $368.27 \mathrm{~kJ} / \mathrm{mol}$, 表明 $\mathrm{HgSO}_{4}, \mathrm{HgO}$ 和录离子能够促进硫 酸气溶胶自发形成.

从以上分析发现，采及其化合物对硫酸气溶胶成核 影响是不一致的, $\mathrm{HgSO}_{4}, \mathrm{HgO}$ 和录离子能够促进硫酸气 溶胶形成, $\mathrm{HgSO}_{4}$ 和 $\mathrm{HgO}$ 都属于极性分子, 采离子荷电, 而 $\mathrm{Hg}, \mathrm{Hg}_{2} \mathrm{Cl}_{2}$ 和 $\mathrm{HgCl}_{2}$ 对硫酸气溶胶形成影响不大, 是 由于它们整体是非极性的, 尽管 $\mathrm{Hg}_{2} \mathrm{Cl}_{2}$ 和 $\mathrm{HgCl}_{2}$ 分子中 有 $\mathrm{Hg}-\mathrm{Cl}$ 极性键, 但由于分子对称性, 极性抵消, 整个 分子没有极性. 由此可知, 采及其化合物对硫酸气溶胶 成核的影响, 与采化合物分子的极性和录原子是否荷电 密切相关.

为了探讨在标准大气压为 $0.1 \mathrm{MPa}$ 下, 大气对流层

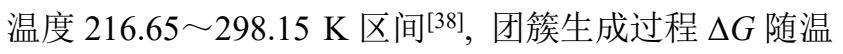
度变化, 我们计算了对流层不同温度下 8 种团簇的 Gibbs 自由能变, 结果如图 4, 详细数据见 SI 表 S13.

从图 4 和 SI 表 S13 中可以发现, 在大气对流层温 度区间, 8 种团族生成过程 $\Delta G$ 均小于 0 , 表明在对流层 环境下这些团簇均能够自发形成, 且从图 4 可发现录的 极性化合物 $\mathrm{HgO}$ 和 $\mathrm{HgSO}_{4}$ 以及 $\mathrm{Hg}_{2}{ }^{2+}$ 和 $\mathrm{Hg}^{2+}$ (黑色)参 与生成团簇时, 都能促进硫酸气溶胶形成, 说明本工作 得出的结论在对流层温度范围同样成立.

\section{3 永矿地区大气中颗粒物观测}

本工作使用量子化学组合方法研究了录及其化合
物对硫酸气溶胶成核的影响，得出非常重要的结论. 同 时我们希望通过对含录地区和不含录地区大气中的颗 粒物进行平行观测, 其结果能对本工作理论计算得出的 结论提供实验支撑.

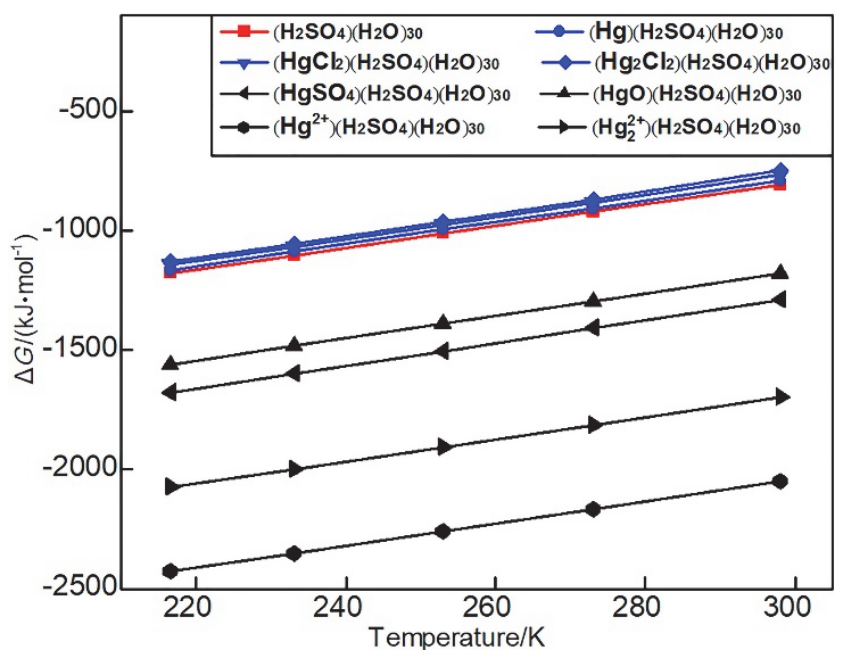

图 48 种团簇生成过程的 $\Delta G$ 随温度变化关系

Figure 4 The relationship between $\Delta G$ and temperature of 8 cluster formation processes

我们选择 $\mathrm{A}, \mathrm{B}, \mathrm{C}$ 和 $\mathrm{D}$ 地进行观测. 其中某省 $\mathrm{A}$ 地 的录矿储量和产量均较高, 在录矿开采过程中排放大量 炼录炉渣和尾矿废渣, 且早期未对这些录矿渣进行处理, 与之对比的 $\mathrm{B}$ 地没有丞矿. 另一省 $\mathrm{C}$ 地也有丰富的录矿 资源, 且目前仍在大规模进行采矿开采、冶炼和加工, 与其对比的 D 地无录矿. 这些有录矿的地区产生的大量 三废排放到环境中, 即使经过处理, 仍可能对周围的大 气环境造成不同程度的录污染.

通过观测 A 地和 B 地(两地距离约 $99.4 \mathrm{~km}$ ) 2019 年 1 月至 2020 年 10 月大气环境中各污染物含量, $\mathrm{A}$ 和 $\mathrm{B}$ 两地大气中的 $\mathrm{SO}_{2}$ 平均含量仅相差 $0.18 \mu \mathrm{g} / \mathrm{m}^{3}$ (具体数值 见 SI 表 S14 S36), 但由图 5(a)发现, A 地的 $\mathrm{PM}_{10}$ 和 $\mathrm{PM}_{2.5}$ 浓度均大于或等于 $\mathrm{B}$ 地, 尤其冬季相差最为明显. 相差最大的为 2019 年 1 月, 其两地 $\mathrm{PM}_{10}$ 和 $\mathrm{PM}_{2.5}$ 浓度 分别相差达 49 和 $41 \mu \mathrm{g} / \mathrm{m}^{3}$ (具体数值见 SI 表 S37, S38). 由图 5(b)中 C 地和 D 地(两地距离约 $304.8 \mathrm{~km}$ )大气中污 染物含量可以看到，除 2019 年 5 月以外, C 地大气中的 $\mathrm{PM}_{10}$ 浓度均大于或等于 $\mathrm{D}$ 地, 同样冬季相差较大. 两地 $\mathrm{PM}_{2.5}$ 浓度虽相差不太明显, 但整体上仍是 $\mathrm{C}$ 地高于 $\mathrm{D}$ 地.

综上, 在 $\mathrm{SO}_{2}$ 浓度相近的情况下, 丞矿地区的 $\mathrm{PM}_{10}$ 和 $\mathrm{PM}_{2.5}$ 浓度高于某些非永矿地区, 说明录的存在与颗 粒物的浓度呈正相关. 虽然硫和录仅为影响大气中颗粒 物浓度的部分因素, 但通过观测得出的结果仍能较好地 支持本工作理论计算得出的结论，即在录、硫复合污染 环境下, 丞的某些化合物能够促进硫酸气溶胶的形成. 


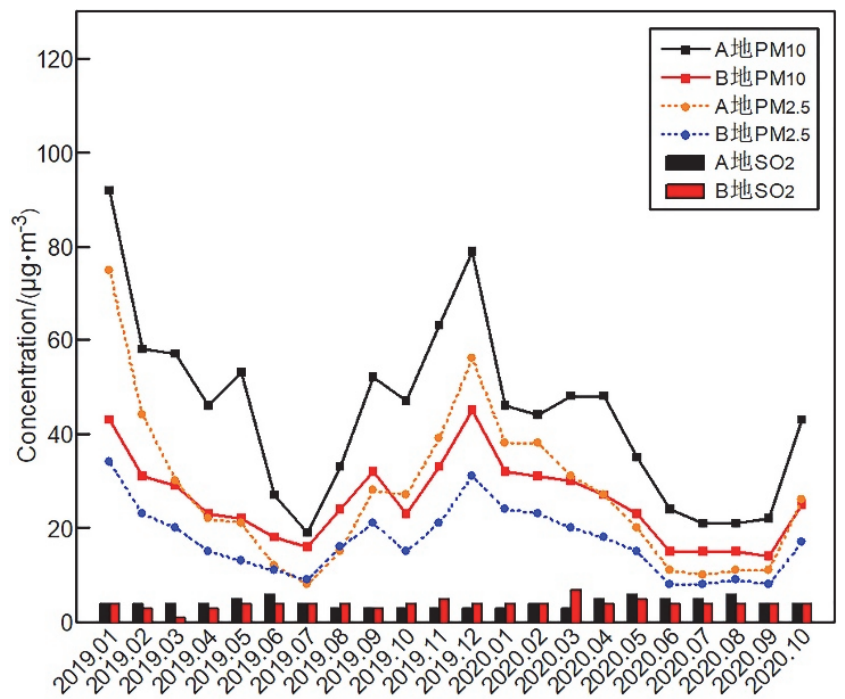

(a)

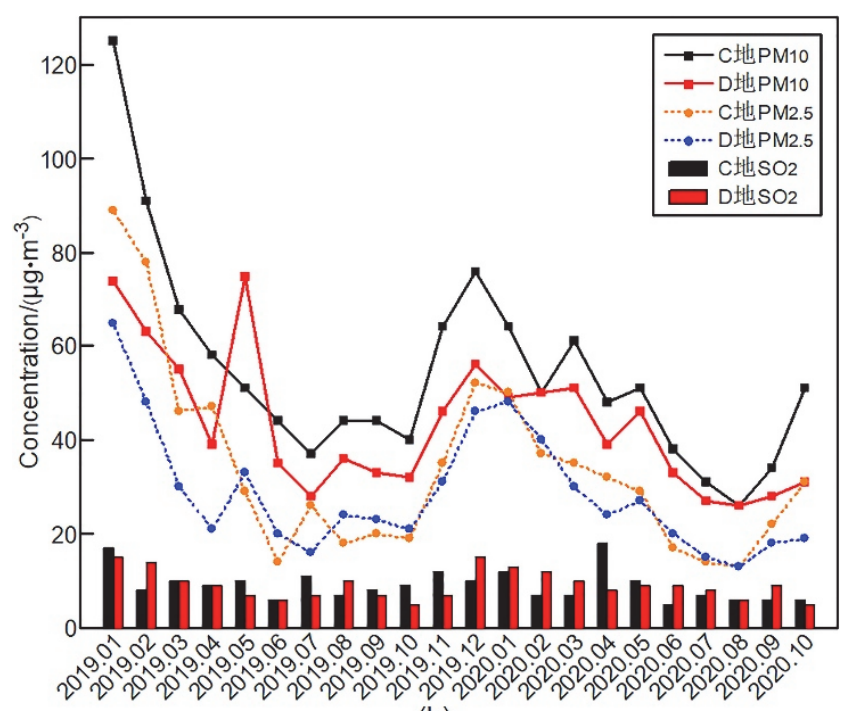

(b)

图 52019 年 01 月 2020 年 10 月 $\mathrm{A}$ 地与 $\mathrm{B}$ 地(a), C 地与 D 地(b)大 气中 $\mathrm{PM}_{10}$ 和 $\mathrm{PM}_{2.5}$ 浓度变化

Figure 5 Changes in atmospheric PM10 and PM2.5 concentrations in A and B place (a), C and D place (b) from January 2019 to October 2020

\section{4 结论}

本工作建立了一种使用 Molclus Genmer 生成团簇 初始结构、自洽紧束缚 GFN2-xTB 方法预优化、Molclus Isostat 去重、B3PW91-D3(BJ) 重新优化团簇结构、 $\omega B 97 M-D 3(B J)$ 校正结合能的组合计算方法. 从 8 种团 簇 240 万个初始结构中篎选出生成过程 Gibbs 自由能变 大的团簇序列及其对应的结合能和热力学函数.

计算结果揭示: $\mathrm{HgO}$ 和 $\mathrm{HgSO}_{4}$ 等录的极性化合物以 及 $\mathrm{Hg}_{2}{ }^{2+}$ 和 $\mathrm{Hg}^{2+}$ 能够促进硫酸气溶胶形成. 而 $\mathrm{Hg}$ 以及 无极性的 $\mathrm{Hg}_{2} \mathrm{Cl}_{2}$ 和 $\mathrm{HgCl}_{2}$ 对硫酸气溶胶成核的影响较 小. 这一结论对区域含硫、采复合大气污染环境下，硫 酸气溶胶成核机制的实验观测提供有价值的预测.

\section{References}

[1] Sipilä, M.; Berndt, T.; Petäjä, T.; Brus, D.; Vanhanen, J.; Stratmann, F.; Patokoski, J.; Mauldin, R. L.; Hyvärinen, A. P.; Lihavainen, H.; Kulmala, M. Science 2010, 327, 1243.

[2] Asaduzzaman, A.; Riccardi, D.; Afaneh, A. T.; Cooper, S. J.; Smith, J. C.; Wang, F.; Parks, J. M.; Schreckenbach, G. Acc. Chem. Res. 2019, 52, 379 .

[3] Hong, Q.; Xie, Z.; Liu, C.; Wang, F.; Xie, P.; Kang, H.; Xu, J.; Wang, J.; Wu, F.; He, P.; Mou, F.; Fan, S.; Dong, Y.; Zhan, H.; Yu, X.; Chi, X.; Liu, J. Atmos. Chem. Phys. 2016, 16, 13807.

[4] Li, L.; Zhang, Y. Y.; Jiao, C. Y.; Yao, Y. W.; Zhang, H.; Tian, Y. M. Environ. Monit. China 2019, 35, 40 (in Chinese). (李亮, 张艳艳，焦 聪颖, 姚雅伟, 张辉, 田英明, 中国环境监测, 2019, 35, 40).

[5] Doyle, G. J. J. Chem. Phys. 1961, 35, 795.

[6] Jaecker, V. A.; Mirabel, P. Atmos. Environ. 1989, 23, 2053.

[7] Sucarrat, M. T.; Francisco, J. S.; Anglada, J. M. J. Am. Chem. Soc. 2012, 134, 20632.

[8] Kildgaard, J. V.; Mikkelsen, K. V.; Bilde, M.; Elm, J. J. Phys. Chem. A 2018, 122, 5026.

[9] Humphries, R. S.; Schofield, R.; Keywood, M. D.; Ward, J.; Pierce, J. R.; Gionfriddo, C. M.; Tate, M. T.; Krabbenhoft, D. P.; Galbally, I. E.; Molloy, S. B.; Klekociuk, A. R.; Johnston, P. V.; Kreher, K.; Thomas, A. J.; Robinson, A. D.; Harris, N. R. P.; Johnson, R.; Wilson, S. R. Atmos. Chem. Phys. 2015, 15, 13339.

[10] Lu, T. Molclus program, Version 1.8.7, Beijing Kein Research Center for Natural Science, China, 2018, http://www.keinsci.com/research/molclus.html

[11] Bannwarth, C.; Ehlert, S.; Grimme, S. J. Chem. Theory Comput. 2019, 15, 1652 .

[12] Tsuzuki, S.; Honda, K.; Uchimaru, T.; Mikami, M.; Tanabe, K. J. Am Chem. Soc. 2002, 124, 104

[13] Hu, M.; Shang, D. J.; Guo, S.; Wu, Z. J. Acta Chim. Sinica 2016, 74, 385 (in Chinese). (胡敏, 尚冬杰, 郭松, 吴志军, 化学学报, 2016, 74, 385).

[14] Elm, J.; Passananti, M.; Kurtén, T.; Vehkamäki, H. J. Phys. Chem. A 2017, 121, 6155.

[15] Yang, P.; Ye, Z. L.; Jiang, G. Y.; Li, Z.; Ding, C. F.; Hou, H. Q. Acta Chim. Sinica 2009, 67, 2031 (in Chinese). (杨鹏, 叶招莲, 蒋公羽, 李周, 丁传凡, 侯惠奇, 化学学报, 2009, 67, 2031).

[16] Lu, Q.; Luo, Q.; Huang, S.; Li, Y.; Wan, J. J. Chem. Phys. A 2016, $120,4560$.

[17] Lu, Q.; Luo, Q.; Huang, S.; Li, Y. Phys. Chem. Chem. Phys. 2017, 19, 28434.

[18] Rasmussen, F. R.; Besel, V.; Mikkelsen, K. V.; Bilde, M.; Elm, J. J. Phys. Chem. A 2020, 124, 5253.

[19] Dohm, S.; Bursch, M.; Hansen, A.; Grimme, S. J. Chem. Theory Comput. 2020, 16, 2002.

[20] Myllys, N.; Olenius, T.; Kurtén, T.; Vehkamäki, H.; Riipinen, I.; Elm, J. J. Phys. Chem. A 2017, 121, 4812.

[21] Becke, A. D. J. Chem. Phys. 1993, 98, 5648

[22] Perdew, J. P.; Wang, Y. Phys. Rev. 1992, 45, 13244.

[23] Amaro-Estrada, J. I.; Maron, L.; Ramirez-Solis, A. Phys. Chem. Chem. Phys. 2014, 16, 8455.

[24] Castro, L.; Dommergue, A.; Renard, A.; Ferrari, C.; Ramirez-Solis, A.; Maron, L. Phys. Chem. Chem. Phys. 2011, 13, 16772.

[25] Yoo, S.; Apra, E.; Zeng, X. C.; Xantheas, S. S. J. Phys. Chem. Lett. 2010, 1, 3122 .

[26] Neese, F.; Hansen, A.; Liakos, D. G. J. Chem. Phys. 2009, 131 064103.

[27] He, Y.; Wang, Y. B. Acta Phys.-Chim. Sin. 2017, 33, 1149 (in Chinese). (何禹, 王一波, 物理化学学报, 2017, 33, 1149)

[28] Najibi, A.; Goerigk, L. J. Chem. Theory Comput. 2018, 14, 5725.

[29] Mardirossian, N.; Pestana, L. R.; Womack, J. C.; Skylaris, C. K.; Head-Gordon, T.; Head-Gordon, M. J. Phys. Chem. Lett. 2017, 8, 35.

[30] Mardirossian, N.; Gordon, M. H. J. Chem. Phys. 2016, 144, 214110.

[31] Boys, S. F.; Bernardi, F. Mol. Phys. 1970, 19, 553.

[32] Kruse, H.; Grimme, S. J. Chem. Phys. 2012, 136, 154101.

[33] Grimme, S. Growing String Method, xTB Documentation, 2019, https://xtb-docs.readthedocs.io/en/latest/gsm.html.

[34] Frisch, M. J.; Trucks, G. W.; Schlegel, H. B.; Scuseria, G. E.; Robb, M. A.; Cheeseman, J. R.; Scalmani, G.; Barone, V.; Mennucci, B.; Petersson, G. A.; Nakatsuji, H.; Caricato, M.; Li, X.; Hratchian, H. P.; Izmaylov, A. F.; Bloino, J.; Zheng, G.; Sonnenberg, J. L.; Hada, M.; Ehara, M.; Toyota, K.; Fukuda, R.; Hasegawa, J.; Ishida, M.; 
Nakajima, T.; Honda, Y.; Kitao, O.; Nakai, H.; Vreven, T.; Montgomery Jr., J. A.; Peralta, J. E.; Ogliaro, F.; Bearpark, M.; Heyd, J. J.; Brothers, E.; Kudin, K. N.; Staroverov, V. N.; Keith, T.; Kobayashi, R.; Normand, J.; Raghavachari, K.; Rendell, A.; Burant, J. C.; Iyengar, S. S.; Tomasi, J.; Cossi, M.; Rega, N.; Millam, J. M.; Klene, M.; Knox, J. E.; Cross, J. B.; Bakken, V.; Adamo, C.; Jaramillo, J.; Gomperts, R.; Stratmann, R. E.; Yazyev, O.; Austin, A. J.; Cammi, R.; Pomelli, C.; Ochterski, J. W.; Martin, R. L.; Morokuma, K.; Zakrzewski, V. G.; Voth, G. A.; Salvador, P.; Dannenberg, J. J.; Dapprich, S.; Daniels, A. D.; Farkas, O.; Foresman, J. B.; Ortiz, J. V.; Cioslowski, J.; Fox, D. J. Gaussian 16, Revision B. 01, Gaussian, Inc., Wallingford CT, 2016.

[35] Neese, F., Software update: the ORCA program system, version 4.2 WIREs: Comput. Mol. Sci. 2018, 8, e1327.

[36] Werner, H. J.; Knowles, P. J.; Knizia, G.; Manby, F. R.; Schütz, M.; Celani, P.; Györffy, W.; Kats, D.; Korona, T.; Lindh, R.;
Mitrushenkov, A.; Rauhut, G.; Shamasundar, K. R.; Adler, T. B.; Amos, R. D.; Bennie, S. J.; Bernhardsson, A.; Berning, A.; Cooper, D. L.; Deegan, M. J. O.; Dobbyn, A. J.; Eckert, F.; Goll, E.; Hampel, C.; Hesselmann, A.; Hetzer, G.; Hrenar, T.; Jansen, G.; Köppl, C.; Lee, S. J. R.; Liu, Y.; Lloyd, A. W.; Ma, Q.; Mata, R. A.; May, A. J.; McNicholas, S. J.; Meyer, W.; Miller III, T. F.; Mura, M. E.; Nicklass, A.; O’Neill, D. P.; Palmieri, P.; Peng, D.; Pflüger, K.; Pitzer, R.; Reiher, M.; Shiozaki, T.; Stoll, H.; Stone, A. J.; Tarroni, R.; Thorsteinsson, T.; Wang, M.; Welborn, M. MOLPRO, version 2018, a package of ab initio programs. http://www. molpro.net.

[37] Yang, Z. Z.; Meng, X. F.; Zhao, D. X.; Gong, L. D. Acta Chim. Sinica 2009, 67, 2074 (in Chinese). (杨忠志, 孟祥凤, 赵东霞, 宫利东, 化学学报, 2009, 67, 2074).

[38] Temelso, B.; Morrell, T. E.; Shields, R. M.; Allodi, M. A.; Wood, E. K.; Kirschner, K. N.; Castonguay, T. C.; Archer, K. A.; Shields, G. C. J. Phys. Chem. A 2012, 116, 2209.

(Cheng, B.) 